\title{
Herd Immunity or Community Immunity
}

\author{
Abdulkareem Salman Khudhair (M.N.S) ${ }^{*}$
}

College of Nursing, University of Basra Iraq

DOI: $10.36347 /$ sjmcr.2020.v08i04.026

| Received: 15.04.2020 | Accepted: 23.04.2020 | Published: 30.04.2020

*Corresponding author: Abdulkareem Salman Khudhair (M.N.S)

\section{Abstract}

Herd immunity is a form of indirect protection against an infectious disease, and occurs when a large proportion of the community acquires immunity to a particular enemy, either from pre-infection or vaccination, which provides protection to individuals who are not immune to the disease [1]. It is a type of immunity that occurs when a vaccination given to a large part of the population (or herd) provides a measure of protection to individuals who are not immune. The theory of herd immunity suggests that in the case of individual-transmitted infectious diseases, chains of infection are likely to be blocked when large numbers of the population are immune to diseases [2]. Herd immunity was first used in 1923. It was recognized as a natural phenomenon in the 1930s, when it was observed that after a large number of children developed measles immunity, the number of new infections decreased temporarily, especially among children at risk. Mass insemination to cause herd immunity has been common ever since, and has proven successful in preventing 3the spread of many infectious diseases. Opposition to vaccination challenged herd immunity, allowing preventable diseases to continue to occur or re-emerge in communities with inadequate vaccination rates [3]. If a large proportion of the population is immune to a particular disease, it helps these people not transmit the disease, so chains of infection are likely to stop, stopping or slowing the spread of the disease. The higher the proportion of individuals who are immune in society, the less likely they are to mix with people who are carriers of the disease, which helps protect them from infection [4]. The human body fights infectious diseases through the immune system when the immune system is exposed to a new enemy - a virus - it deals with it, and if the person lives and recovers, the immune system develops a memory of this invader, so that if exposed to the virus in the future he can fight it easily [5].

Keyword: Herd, immunity, infection, vaccination

Copyright @ 2020: This is an open-access article distributed under the terms of the Creative Commons Attribution license which permits unrestricted use, distribution, and reproduction in any medium for non-commercial use (NonCommercial, or CC-BY-NC) provided the original author and source are credited.

\section{Protecting The Unimmunized}

Charlotte Cleverley-Bessman, whose four limbs were partially amputated at the age of seven months due to meningococcal disease, a transferable disease that can be reduced through herd immunity. Some individuals cannot develop immunity after medical insemination or for medical reasons that cannot be vaccinated. Newborns are considered too young to receive many vaccines, either for safety reasons or because their negative immunity makes the vaccine ineffective. Some individuals may lose the immunity they had previously, such as AIDS, lymphoma, leukemia, bone marrow cancer, spleen weakness, chemotherapy or radiation therapy, and vaccines may be of no use due to their immunodeficiency [6].

High levels of immunity in a specific age group may lead to herd immunity for other age groups. Vaccinating adults against whooping cough reduces the incidence of whooping cough in young infants who cannot be vaccinated and who are considered to be at higher risk of complications. This is particularly important for family members, who represent most cases of transmission to young infants. In the same way, children receiving pneumococcal vaccines reduce pneumococcal disease among younger, unvaccinated siblings[7].

\section{Evolutionary pressure}

Herd immunity itself exerts evolutionary pressure on certain viruses, affecting viral development, encouraging the production of new strains, referred to as escape mutants, where they are able to escape from herd immunity and spread more easily. At the molecular level, viruses escape herd immunity through antigens, which occur when mutations accumulate in a part of the virus's genome that encodes the virus's surcharge, usually a protein from the viruses vile, leading to a change in viral infection. 
Alternatively, reclassification of separated viral genome parts or antigen (which is more common when more strains within the circulatory system) may end in new serotypes. When either of those occurs, memory $\mathrm{T}$ cells don't recognize the virus, so individuals don't seem to be resistant to the dominant known strain. For both influenza and norovirus, epidemics temporarily stimulate herd immunity until a replacement dominant strain emerges, causing successive waves [8].

\section{Replacing the seropattern}

The replacement or conversion of the seropattern may occur if the prevalence of a specific serotype decreases due to high levels of immunity, allowing it to be replaced by other serotypes. Primary vaccines against streptococcus significantly reduced the nasopharyngeal transmission of vaccine serotypes (VTs), including antibiotic-resistant species, to be fully compensated by increased transmission of non-vaccine serotypes (NVTs). This did not lead to a proportionate increase in the incidence of the disease, as non-vaccine serotypes (NVTs) were less intrusive than the serotypes of the Vaccine (VTs). Since then, pneumococcal vaccines that provide protection against emerging serotypes have been introduced and have succeeded in the event from their appearance. The possibility of serotypes mutating still exists, so further strategies to deal with this include expanding the coverage of vaccine serotypes and developing vaccines that use either dead full cells, containing more surface antigens, or proteins found in many serotypes[9].

\section{Eradication of diseases}

If the herd's immunity is confirmed and maintained in a population for a sufficient period, the disease will certainly be eliminated, i.e. no more endemic transmissions will occur. If the disease is eliminated worldwide and the number of cases is permanently reduced to zero, eradication can be declared, and therefore eradication can be considered a final effect or the ultimate outcome of public health initiatives aimed at controlling the spread of infectious diseases.

There are several benefits to the eradication of the disease, including the eradication of the disease and its ability to cause death, the provision of funds for individuals, health care providers, and governments, and the empowerment of resources used to control the disease for use elsewhere. To date, two diseases have been eliminated using herd immunity and pollination, namely, cow plague and smallpox. Efforts are being made to eradicate polio based on herd immunity, although civil disorders and lack of confidence in modern medicine have made this difficult. Compulsory vaccination may be useful for eradication efforts if there are not enough vaccinated individuals [10].

\section{Vaccination}

The main way to boost immune levels in a population is through vaccination. Vaccination was originally based on the observation that female workers in cow milking who had been exposed to chickenpox were anti-smallpox, so the practice of vaccinating people with chickenpox began as a way to prevent smallpox. Well-developed vaccines provide protection in a much safer way than the natural disease because vaccines usually do not cause diseases, they protect against and adverse effects are much less common than the complications caused by natural infections. The immune system does not distinguish between natural infections and vaccines and active response to both, so the immunity updated through vaccination is similar to what would have occurred when the disease was infected and cured. To achieve herd immunity through vaccination, vaccine manufacturers aim to produce vaccines with low failure rates and policymakers aim to encourage their use. After the successful introduction and significant spread of the vaccine, a sharp decrease in the incidence of protected diseases, as well as the number of hospitalizations and deaths caused by these diseases, was observed [11].

\section{REFERENCES}

1. https://web.archive.org/web/20161223192137/http: //cid.oxfordjournals.org:80/content.

2. https://elaph.com/Web/healthscience/2020/03/1285245.html.

3. https://ar.wikipedia.org/wiki/

4. https://web.archive.org/web/20190610101054/https ://books.google.com/books?

5. https://web.archive.org/web/20190612183021/https ://pediatrics.aappublications.org

6. https://web.archive.org/web/20170424022811/https ://books.google.com.

7. https://www.ncbi.nlm.nih.gov/pmc/articles/PMC40 83064/

8. https://www.ncbi.nlm.nih.gov/pmc/articles/PMC37 82273/

9. https://www.ncbi.nlm.nih.gov/pmc/articles/PMC34 78140/

10. https://web.archive.org/web/20160624072838/https ://books.google.com

11. https://pediatrics.aappublications.org/content/134/2 /325. 